\section{Osteonecrosis de los maxilares asociada a medicamentos: revisión de la literatura y propuesta para la prevención y manejo}

\author{
CAMILA FONCEA ${ }^{1, \mathrm{a}, \mathrm{d}}$, KRISTINE VON BISCHHOFFSHAUSEN $^{1, \mathrm{a}, \mathrm{e}}$, \\ CRISTIÁN TEUBER ${ }^{1, \mathrm{a}, \mathrm{d}}$, HERNÁN RAMÍREZ ${ }^{1, \mathrm{a}, \mathrm{c}}$, IGNACIO GOÑI ${ }^{1, \mathrm{~b}}$, \\ CESAR SÁNCHEZ ${ }^{2, \mathrm{~g}}$, IGNACIO N. RETAMAL ${ }^{3, \mathrm{a}, \mathrm{f}}$, ALEX VARGAS $^{1, \mathrm{a}, \mathrm{c}}$
}

\section{Osteonecrosis of the jaws}

Medication-related osteonecrosis of the jaw is a disease where there is necrotic bone exposed or that can be explored by means of a fistula in the maxillofacial region. It has been associated with the use Biphosphonates and denosumab for osteoporosis. Although its etiology is unclear, it may be related to a decrease in bone turnover produced by these drugs, rendering the bone more prone to generate cell necrosis during invasive dental procedures, especially in the posterior region of the jaw. There is no consensus about the prevention and treatment of this condition. The aim of this paper is to present a review of the literature with the main characteristics of osteonecrosis of the jaws associated with drugs, together with a proposal for prevention and treatment for these patients.

(Rev Med Chile 2020; 148: 983-991)

Key words: Osteonecrosis; Angiogenesis Inhibitors; Bisphosphonate-Associated Osteonecrosis of the Jaw; Bone Density Conservation Agents; Diphosphonates.
'Departamento de Cirugía Oncológica y Maxilofacial, Facultad de Medicina, Pontificia Universidad Católica de Chile. Santiago, Chile.

2Departamento de Hemato-Oncología, Facultad de Medicina, Pontificia Universidad Católica de Chile. Santiago, Chile.

${ }^{3}$ Facultad de Odontología, Universidad de Los Andes. Santiago, Chile.

${ }^{a}$ Cirujano Dentista.

${ }^{\text {b}}$ Cirujano Cabeza y Cuello.

'Cirujano Maxilofacial.

${ }^{d}$ Residente Cirugía Maxilofacial.

ePasante Cirugía Maxilofacial.

fDoctor en Ciencias.

9Oncólogo Médico.

Trabajo no recibió financiamiento. Los autores declaran no tener conflictos de interés.

Recibido el 14 de junio de 2019, aceptado el 13 de marzo de 2020.

Correspondencia a:

Dr. Alex Vargas

Departamento de Cirugía Oncológica y

Maxilofacial

Facultad de Medicina, Pontificia

Universidad Católica de Chile.

Av. Diagonal Paraguay 362, $3^{\circ}$ Piso,

Santiago Centro, RM, Chile.

avargasi@uc.cl
L La osteonecrosis de los maxilares (ONM) asociada a medicamentos, también denominada MRONJ por sus siglas en inglés, se define como la presencia de hueso necrótico expuesto o que puede ser explorado mediante una fístula, en el territorio maxilofacial, que se mantiene durante un período mínimo de 8 semanas, sin antecedente de radioterapia en la zona. Esta condición es infrecuente y se ha visto asociada a dos grupos de medicamentos: los antiresortivos y los antiangiogénicos ${ }^{1,2}$.

Los fármacos antiresortivos, o agentes modificadores del metabolismo óseo, están indicados en enfermedades con aumento de la resorción ósea, como osteoporosis, hipercalcemia maligna, metástasis óseas de tumores sólidos o mieloma múltiple. Compuestos como los bifosfonatos o de- nosumab se incluyen en este grupo de fármacos ${ }^{1,3}$. Los medicamentos antiangiogénicos son utilizados para el tratamiento de tumores gastrointestinales, carcinomas renales, tumores neuroendocrinos y otras enfermedades malignas; actúan inhibiendo la formación de vasos sanguíneos e interrumpiendo la cascada de señalización que resulta en la angiogénesis. Sunitib o bevacizumab son ejemplos de este grupo ${ }^{1}$.

Ambos grupos de medicamentos han demostrado ser agentes farmacológicos efectivos, pero desde el año 2003 se han reportado informes de ONM en pacientes tratados con este tipo de drogas ${ }^{4}$. Si bien la ONM es una complicación infrecuente, se asocia a una morbilidad importante, pudiendo afectar significativamente la calidad de vida del paciente ${ }^{5,6}$. 
El objetivo del siguiente trabajo es realizar una revisión de la literatura y proponer un protocolo para la prevención y tratamiento de la ONM asociada a medicamentos. Para esto, se realizó una revisión bibliográfica en las bases de datos PubMed y Epistemónikos, utilizando los términos de búsqueda "osteonecrosis de los maxilares" "bifosfonatos" "terapia antiresortiva" "terapia antiangiogénica". Se incluyeron tanto artículos en inglés como en español publicados entre el año 2005 y 2018. La selección de los artículos fue realizada de común acuerdo entre dos revisores, según la relevancia y aportes en medidas de prevención y manejo de la ONM.

\section{Incidencia}

La ONM afecta generalmente a pacientes de edad avanzada ${ }^{7}$, con tasas de incidencia que van de $0,94 \%$ a $10 \%$ en distintos grupos de población con diferentes regímenes de drogas, siendo las extracciones dentales u otro procedimiento dental invasivo el principal factor desencadenante de la enfermedad ${ }^{8,9}$.

Se ha observado que los bifosfonatos endovenosos son los fármacos más frecuentemente aso- ciados al desarrollo de ONM, con una incidencia que va de $0,8 \%$ a $12 \%{ }^{9}$. Esta incidencia aumenta en pacientes en tratamiento por mieloma múltiple y cáncer de mama metastásico ${ }^{10,11}$. La metástasis se presenta como un predictor de riesgo importante ${ }^{12}$.

Respecto a la ubicación, la ONM se presenta con mayor frecuencia en la mandíbula (entre $65 \%$ y $95,24 \%$ de los casos), especialmente en la zona lingual del reborde alveolar de premolares y molares inferiores ${ }^{10}$. Se cree que esta zona es más propensa debido al trauma masticatorio frecuente, lo que pudiese causar daño en la mucosa y periostio, conduciendo a la necrosis del hueso ${ }^{10}$.

\section{Presentación clínica}

El diagnóstico de ONM se realiza por la identificación clínica de una o varias úlceras con hueso expuesto de aspecto necrótico en la región de los maxilares ${ }^{13}$. El diagnóstico diferencial incluye enfermedad periodontal, osteomielitis, sinusitis, osteo-radionecrosis y tumores óseos primarios o metástasis ${ }^{14}$. Se describen distintos estadios clínicos, de acuerdo a la severidad de la patología $(\text { Tabla } 1)^{1,2,15}$.

Tabla 1. Clasificación de estadios clínicos de la osteonecrosis de los maxilares según sus distintas características $^{1-2,7}$

\begin{tabular}{|c|c|c|}
\hline Estadio & $\begin{array}{l}\text { American Association of Oral and } \\
\text { Maxillofacial Surgeons (AAOMS) } 2014\end{array}$ & $\begin{array}{c}\text { International Task Force on Osteonecrosis } \\
\text { of the Jaw } 2015\end{array}$ \\
\hline 0 & $\begin{array}{l}\text { Paciente sin evidencia clínica de hueso necrótico, } \\
\text { que presenta síntomas no específicos o hallazgos } \\
\text { radiográficos que no permiten confirmar diagnóstico }\end{array}$ & - \\
\hline 1 & $\begin{array}{l}\text { Definido como la exposición y necrosis del hueso o la } \\
\text { presencia de una fístula comunicada hacia el hueso } \\
\text { en pacientes asintomáticos y que no tienen signos } \\
\text { evidentes de infección }\end{array}$ & $\begin{array}{l}\text { Asintomático, hueso expuesto en mandíbula o } \\
\text { maxilar, con tejido blando adyacente o regional sin } \\
\text { evidencia de inflamación ni de signos de infección } \\
\text { secundaria }\end{array}$ \\
\hline 2 & $\begin{array}{l}\text { Presencia de hueso necrótico expuesto o de una fís- } \\
\text { tula comunicada al hueso, en pacientes con síntomas } \\
\text { y signos evidentes de infección }\end{array}$ & $\begin{array}{l}\text { Dolor, hueso expuesto en mandíbula o maxilar, con } \\
\text { tejido blando adyacente o regional inflamado o con } \\
\text { signos de infección secundaria }\end{array}$ \\
\hline 3 & $\begin{array}{l}\text { Presencia de hueso necrótico expuesto o fístula co- } \\
\text { municada al hueso, en pacientes con dolor, infección } \\
\text { y uno o más de los siguientes elementos: exposición } \\
\text { de hueso necrótico más allá de la región del hueso } \\
\text { alveolar (por ejemplo: el borde inferior o rama man- } \\
\text { dibular o seno maxilar), fractura patológica, fístula } \\
\text { extraoral, comunicación oroantral u oronasal y/o } \\
\text { osteolísis extendiéndose al borde mandibular inferior } \\
\text { o al piso del seno maxilar }\end{array}$ & $\begin{array}{l}\text { Dolor, hueso expuesto en mandíbula o maxilar, con } \\
\text { tejido blando adyacente o regional inflamado o con } \\
\text { signos de infección secundaria, presencia de fístula } \\
\text { extraoral u oroantral o evidencia radiográfica de } \\
\text { osteolísis extendiéndose al borde inferior de la man- } \\
\text { díbula o al piso del seno maxilar }\end{array}$ \\
\hline
\end{tabular}


La ONM puede permanecer asintomática en aproximadamente $30 \%$ de los pacientes y se identifica sólo por la presencia clínica de hueso expuesto en la cavidad oral ${ }^{14}$. Generalmente, cuando existe sintomatología asociada, es debido al compromiso inflamatorio y/o infeccioso de los tejidos blandos vecinos a la zona necrótica ${ }^{1}$.

Existen hallazgos clínicos y radiográficos que permiten realizar el diagnóstico (Tablas $2 \mathrm{y}$ $3)^{16,17}$. Estos hallazgos son inespecíficos y pueden presentarse en cualquiera de los cuatro estadios. La tomografía computarizada es un examen útil que permite determinar y evaluar la extensión de la ONM en relación con lo observado clínicamente $^{13}$. Histológicamente es posible observar hueso necrótico con lagunas de osteocitos y espacios medulares vacíos, desprovistos de los elementos celulares normales ${ }^{18}$.

\section{Patogenia y factores de riesgo}

Aunque la ONM asociada a medicamentos puede iniciarse espontáneamente, la mayoría de los casos se asocian a tratamientos dentales invasivos o traumatismos locales como prótesis mal ajustadas ${ }^{1,6,19}$. Las extracciones dentales corresponden al factor predisponente más común, aumentando el riesgo de desarrollar ONM en pacientes en tratamiento con bifosfonatos ${ }^{6}$.

Si bien no se ha definido de manera precisa la etiología de la ONM asociada al uso de medicamentos, se ha propuesto como principal responsable la supresión del recambio y remodelación ósea, mediada por el efecto inhibidor sobre los osteoclastos que determina el efecto antiresortivo como acción terapéutica ${ }^{7,20}$. Sin los osteoclastos, no existe una señal para la formación de nuevos osteoblastos y el hueso se vuelve acelular y necrótico, los capilares del hueso involucionan y este se vuelve avascular ${ }^{10}$. Este fenómeno sumado a una injuria de la mucosa puede exponer al medio oral un hueso que ha perdido su capacidad de regeneración $^{20,21}$. La ubicación de la ONM en el territorio oral se explica por el alto rango de remodelación del hueso alveolar maxilar y mandibular, ${ }^{1,4}$.

Otro mecanismo de acción propuesto tiene que ver con la capacidad de inhibición de la angiogénesis, al interferir con la función de las células endoteliales vasculares mediante una reducción de la proliferación, aumento de la apoptosis y
Tabla 2. Principales hallazgos clínicos encontrados en la osteonecrosis de los maxilares ${ }^{1,14,17}$

Hallazgos clínicos
Odontalgia no explicada por causa odontogénica
Dolor sordo que puede irradiarse a la región de la articu-
lación temporomandibular
Dolor sinusal asociado o no a inflamación y/o engrosa-
miento de la mucosa del piso sinusal
Alteración de la función neurosensorial
Movilidad dentaria no asociada a enfermedad periodontal
crónica; y/o fístula periapical o periodontal no asociada a
necrosis pulpar por caries, trauma o restauraciones

Tabla 3. Hallazgos radiográficos encontrados en la osteonecrosis de los maxilares ${ }^{1,2}$

\section{Hallazgos radiográficos}

Pérdida de hueso alveolar o reabsorción no atribuible a enfermedad periodontal crónica

Cambios en el trabeculado óseo y la no formación de hueso nuevo en alvéolos post extracción

Regiones de osteoesclerosis que incluyan el hueso alveolar o que rodeen el hueso basilar y/o engrosamiento o disminución del ligamento periodontal apical o de la lámina dura

disminución de la formación de túbulos de tipo capilar $^{7,20,22}$. Según esta teoría, la inhibición de la proliferación endotelial en los maxilares, conduciría a la pérdida de los vasos sanguíneos y necrosis avascular $^{21}$.

Diversos factores se han visto relacionados con un aumento de riesgo de ONM. Uno de los más importantes es la potencia del fármaco para el caso de los bifosfonatos ${ }^{22}$, siendo el ácido zolendrónico el antiresortivo más frecuentemente relacionado con el desarrollo de $\mathrm{ONM}^{23}$. La vía de administración intravenosa también aumenta el riesgo de desarrollar ONM y favorece su presentación en estadios más avanzados ${ }^{3}$. La duración y frecuencia del tratamiento también se consideran factores de riesgo, especialmente por la larga vida media que pueden tener estos fármacos ${ }^{5,22}$. En relación a los bifosfonatos administrados por vía oral, se ha observado una mayor incidencia de ONM 
en pacientes tratados por un período de tiempo mayor a 3 años ${ }^{5}$. Las extracciones dentales son el procedimiento dental más relacionado con la ONM; sin embargo, la presencia de enfermedad periodontal activa y piezas dentales en mal estado también pueden iniciar una $\mathrm{ONM}^{15,24}$.

\section{Tratamiento y manejo}

El manejo de la ONM asociada a medicamentos es aún controversial. Si bien han sido publicados varios documentos de consenso, la evidencia disponible es insuficiente para generar guías estandarizadas para el tratamiento específico en cada estadio de la enfermedad ${ }^{1,15,25,26}$.

En términos generales, los objetivos del tratamiento son la prevención e identificación precoz de la enfermedad. Una vez instalada, se debiese controlar el dolor y la infección, minimizar la progresión de la necrosis ósea y establecer un ambiente adecuado para la cicatrización de los tejidos $^{6,26}$.

Dentro de las alternativas descritas en la literatura para el manejo de la ONM, se describe el uso de antimicrobianos tópicos y sistémicos, terapia con oxígeno hiperbárico $(\mathrm{OHB})$, cirugía para resecar secuestros óseos, uso de concentrados plaquetarios y la terapia farmacológica combinada de pentoxifilina y vitamina E (PENT-E) ${ }^{6,13,27}$.

En pacientes con pequeñas áreas de $\mathrm{ONM}$, el tratamiento debe ser conservador, indicando antimicrobianos tópicos como colutorios de clorhexidina a $0,12 \%$ y manejo analgésico ${ }^{6,26}$. Cuando existe evidencia de inflamación o infección local, se recomienda el uso de antibióticos de amplio espectro derivados de las penicilinas ${ }^{13,26}$. En pacientes alérgicos a penicilinas se puede indicar metronidazol, quinolonas, clindaminina, doxiciclina o eritromicina ${ }^{6}$.

En pacientes con áreas de ONM expuestas o que no evolucionen satisfactoriamente tras un tratamiento conservador, es necesario intervenir quirúrgicamente para eliminar el área de hueso necrótico y/o los secuestros óseos, independiente del estadio de la enfermedad, además de realizar una biopsia en caso de sospecha de enfermedad metastásica ${ }^{6,13,26}$. Un estudio prospectivo reportó que la resección quirúrgica de los secuestros óseos en conjunto con tratamiento antibiótico con amoxicilina con ácido clavulánico y colutorios de clorhexidina, si bien no logró una curación completa de la enfermedad en todos los casos, generó una mejoría significativa de los síntomas en más de $80 \%$ de los casos de ONM en estadio II y III $^{28}$.

El uso de OHB se ha planteado como una alternativa mínimamente invasiva para el manejo de la ONM. El OHB genera especies reactivas de oxígeno y nitrógeno, que sirven como señal para estimular la angiogénesis y acelerar la tasa de diferenciación de osteoblastos, lo que conduciría a un aumento en la formación de hueso ${ }^{29}$. Clínicamente, el OHB parece ser un complemento útil al tratamiento de la ONM, en particular para los casos más graves, refractarios a tratamiento habitual $^{27}$. Lamentablemente, aún no hay evidencia suficiente para apoyar su uso sobre las técnicas convencionales y los altos costos asociados limitan su indicación de forma rutinaria ${ }^{30}$.

Los concentrados plaquetarios autólogos, como el plasma rico en plaqueta (PRP) o la fibrina rica en plaquetas (PRF), han ganado popularidad en el último tiempo para el tratamiento y prevención de la $\mathrm{ONM}^{31}$. Estos concentrados aportan factores de crecimiento y citoquinas específicas que estimulan la angiogénesis, la diferenciación celular, la producción de colágeno y agentes antiinflamatorios ${ }^{31}$. Una revisión sistemática reportó que el uso de concentrados plaquetarios junto al debridamiento quirúrgico ayuda a la resolución y disminución de los síntomas en pacientes con ONM; y también tendrían un rol en la prevención de la enfermedad, disminuyendo el riesgo de desarrollo de ONM al utilizarlos en el lecho quirúrgico de pacientes que requieran someterse a procedimientos quirúrgicos invasivos como extracciones dentales ${ }^{31}$.

Otra alternativa conservadora, de bajo costo $y$ con resultados prometedores, es el uso de pentoxifilina asociado con vitamina E (PENT-E). Su aplicación fue descrita inicialmente para el tratamiento y prevención de la osteorradionecrosis de los maxilares ${ }^{32}$. La PENT-E es una terapia bien tolerada que ha reportado resultados favorables en la resolución de los síntomas y en la cicatrización ósea y de la mucosa oral en pacientes con $\mathrm{ONM}^{33}$. La pentoxifilina era indicada clásicamente para el tratamiento de la cardiopatía isquémica y claudicación intermitente, ya que mejora el flujo sanguíneo periférico e induce la acción del factor alfa de necrosis antitumoral (anti-TNFa), inhibiendo así la inflamación y disminuyendo la 
fibrosis ${ }^{34}$. La vitamina $\mathrm{E}$ (tocoferol) es un potente antioxidante que reduce el daño causado por los radicales libres, la inflamación y la fibrosis tisular ${ }^{34}$. En pacientes con enfermedad ósea metastásica o mieloma múltiple, diagnosticados con ONM refractaria a los tratamientos habituales, el uso de $400 \mathrm{mg}$ de pentoxifilina y 400 UI de vitamina $\mathrm{E}$, dos veces al día, ha demostrado alivio de los síntomas y neoformación ósea en defectos radiolúcidos de algunos $\operatorname{casos}^{34}$.

\section{Prevención}

La prevención es la mejor y más importante estrategia para manejar la ONM asociada a medicamentos ${ }^{14}$. Antes de iniciar el tratamiento antiresortivo, si la condición sistémica lo permite, es fundamental que el paciente sea evaluado por un odontólogo entrenado o un especialista en cirugía maxilofacial, con el fin de optimizar el estado de salud oral y controlar posibles fuentes de infección $\mathrm{n}^{6,13,26,35}$.

Una vez que se ha iniciado el tratamiento antiresortivo, los controles odontológicos deben mantenerse de forma indefinida, idealmente cada 3 a 6 meses, con el objeto de prevenir y tratar caries y enfermedad periodontal en etapas tempranas, evitando la necesidad de procedimientos quirúrgicos dento-maxilares ${ }^{6,11,14,26}$.

En pacientes que requieran tratamiento odontológico, se recomienda optar por terapias dentales lo menos invasivas posible ${ }^{28}$. La infección dental o periodontal activa debe ser tratada en todos los casos, a pesar del riesgo de desarrollar ONM, con el objeto de evitar las complicaciones asociadas a un proceso infeccioso agudo en el territorio maxilofacial ${ }^{9}$.

El valor de la suspensión de los medicamentos antiresortivos antes de una cirugía dento-maxilar es desconocido y requiere estudios adicionales ${ }^{6,36}$. $\mathrm{Al}$ respecto, es importante considerar, en el caso de los bifosfonatos, que su efecto es prolongado y persistente en el tiempo, llegando a un vida media de hasta 11,2 años para los bifosfonatos intravenosos ${ }^{15}$. La Asociación Dental Americana indica que la decisión de interrumpir la terapia debe ser una decisión médica basada principalmente en el riesgo de eventos esqueléticos secundarios a la baja densidad ósea y no en el posible riesgo de $\mathrm{ONM}^{9,17}$.

\section{Protocolo para prevención y manejo de la osteonecrosis de los maxilares asociada a medicamentos}

En base a los datos disponibles y al trabajo interdisciplinario de los equipos de Cirugía Oncológica y Maxilofacial y Oncología Médica de la Red de Salud UC-Christus, se propone el siguiente protocolo para la prevención y manejo de la ONM asociada a medicamentos, actualmente en uso clínico $^{1,15}$ (Figura 1):

\section{A. Pacientes candidatos a inicio de terapia antiresortiva/antiangiogénica}

1. Evaluación dental previa por un cirujano dentista competente o un cirujano maxilofacial, con un tiempo mínimo de 2 a 3 semanas previo al inicio de la terapia ${ }^{6,13,26,36}$ :

- Evaluación clínica e imaginológica (radiografías panorámica y retro alveolar total, en todos los pacientes), con el objeto de definir la necesidad de realizar procedimientos odontológicos mínimamente invasivos o intervenciones quirúrgicas previo al inicio del tratamiento.

- Estudios imaginológicos complementarios (Tomografías Cone Beam), si es necesario precisar el diagnóstico, pronóstico o manejo de alguna lesión dento-maxilar específica.

- Derivación a otras especialidades según cada caso (cirugía maxilofacial, periodoncia, rehabilitación oral, etc.).

2. Realizar procedimientos quirúrgicos, idealmente 15 a 20 días antes del inicio de la terapia farmacológica antiresortiva/antiangiogénica ${ }^{26}$.

- Intervenciones quirúrgicas dento-maxilares conservadoras, con cierre de heridas por primera intención, remodelación ósea, colgajos libres de tensión y uso adicional de concentrados plaquetarios ${ }^{31}$.

- Terapia antibiótica sistémica, según valoración de riesgo de infección en cada caso.

- Uso de antisépicos tópicos (colutorios de clorhexidina a $0,12 \%$ ) por 14 días o hasta observar cicatrización clínica de la lesión.

- Pentoxifilina $400 \mathrm{mg}+$ Tocoferol (vitamina E) 400 UI cada $12 \mathrm{~h}$, hasta evidencia clínica e imaginológica de cicatrización ósea ${ }^{32,35}$.

3. Educación e instrucción en higiene oral y controles odontológicos periódicos. 


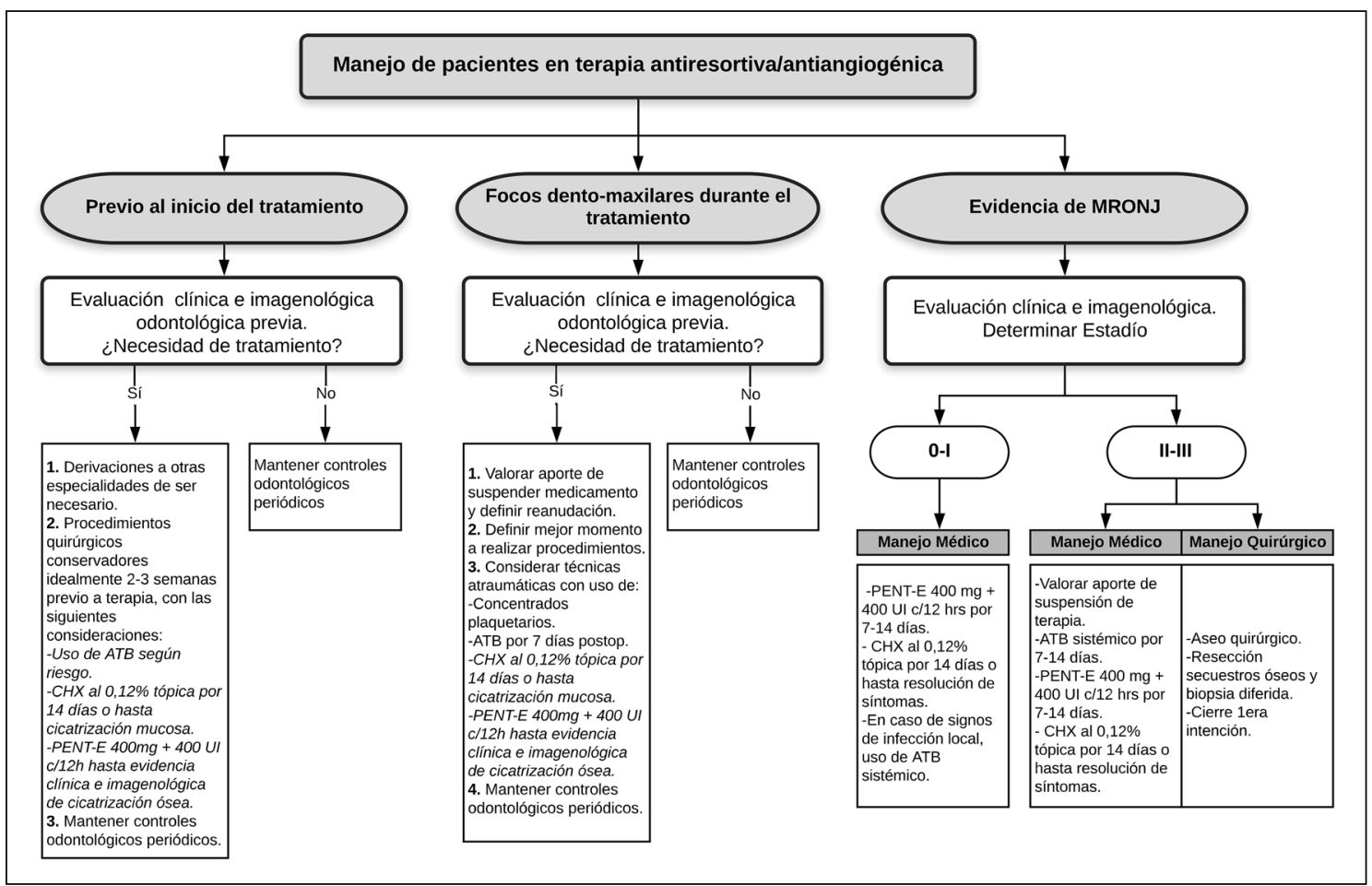

Figura 1. Algoritmo propuesto para prevención y manejo de la osteonecrosis de los maxilares asociada a medicamento.

\section{B. Pacientes en tratamiento antiresortivo/ antiangiogénico que requieran intervenciones quirúrgicas dento-maxilares}

1. Valorar el aporte de la suspensión del medicamento en cada caso ${ }^{26}$.

2. Definir en conjunto con el médico tratante el mejor momento para realizar la intervención.

3. Realizar intervenciones quirúrgicas dento-maxilares conservadoras con cierre mucoso por primera intención, plastía ósea, colgajos libres de tensión y uso adicional de concentrados plaquetarios ${ }^{31}$.

- Terapia antibiótica sistémica (se recomienda el esquema de amoxicilina con ácido clavulánico, $875 / 125 \mathrm{mg}$ cada 12 h como primera línea) por 7 días postoperatorios ${ }^{13}$.

- Uso de antisépicos tópicos (colutorios de clorhexidina a $0,12 \%$ ) hasta observar cicatrización clínica de la lesión.

- Pentoxifilina $400 \mathrm{mg}+$ Tocoferol (vitamina E) 400 UI cada 12 h, hasta evidencia clínica e imaginológica de cicatrización ósea ${ }^{32,35}$.

4. Mantener controles odontológicos periódicos según la valoración de riesgo del paciente de acuerdo al uso, tipo y dosis de fármaco antiresortivo/antiangiogénico, uso concomitante de corticoides, presencia de periodontitis, higiene deficiente, hábito tabáquico o comorbilidades, como desórdenes inmunes, anemias o diabetes mellitus, entre otras ${ }^{26}$.

5. En caso de suspensión del tratamiento antiresortivo/antiangiogénico, definir cuándo reanudar la terapia ${ }^{9,17}$.

\section{Pacientes con evidencia de ONM (de acuerdo a la clasificación de estadios de la American} Association of Oral and Maxillofacial Surgeons $\left.-A A O M S^{I}\right)$ :

1. Estadios 0 y I: Proporcionar tratamiento sintomático y manejar factores locales, como caries y enfermedad periodontal.

- Cuantificación en milímetros del tamaño de la exposición ${ }^{26}$.

- Uso de antisépicos tópicos (colutorios de clorhexidina a $0,12 \%$ ) por al menos 14 días o hasta la resolución de los síntomas ${ }^{6,26}$. 
- Tratamiento antibiótico sistémico para el control de la infección local, cuando sea necesario ${ }^{6,9,13}$.

- Pentoxifilina $400 \mathrm{mg}+$ Tocoferol (vitamina E) 400 UI cada 12 h, hasta la resolución de los síntomas ${ }^{32,35}$.

- Sugerir, si fuera posible, la suspensión del tratamiento antiresortivo/antiangiogénico por parte del especialista que lo prescribió ${ }^{26}$.

- Control cada 7-14 días según evolución ${ }^{26}$.

- El tratamiento quirúrgico no está indicado ${ }^{1,2,6}$.

2. Estadios II y III: Otorgar tratamiento médico-quirúrgico proporcional a las lesiones clínicas que presente el paciente.

- Cuantificación en milímetros del tamaño de la exposición ${ }^{26}$.

- Uso de antisépicos tópicos (colutorios de clorhexidina a $0,12 \%)^{6,26}$.

- Tratamiento antibiótico sistémico (amoxicilina con ácido clavulánico, 875/125 mg cada $12 \mathrm{~h}$ ) por 7 a 14 días ${ }^{1,2,6,13,26}$.

- Pentoxifilina 400mg + Tocoferol (vitamina E) 400 UI cada $12 \mathrm{~h}$, hasta evidencia clínica e imagenológica de resolución del cuadro ${ }^{32,35}$.

- ONM limitada a hueso alveolar:

- Aseo quirúrgico, debridamiento y resección de secuestros óseos mediante intervenciones quirúrgicas mínimamente invasivas ${ }^{1,2,6,26}$.

- Cierre de heridas por primera intención y colgajos libres de tensión.

- Cierre de comunicaciones bucosinusales con colgajo de bola adiposa de Bichat.

- Uso adicional de concentrados plaque$\operatorname{tarios}^{31}$.

- ONM extendida al maxilar o al borde basilar mandibular:

- Aseo quirúrgico, debridamiento y resección extendida de secuestros óseos ${ }^{26}$.

- Osteosíntesis en caso de falta de continuidad ósea mandibular.

- Reconstrucción con colgajo libres micro-vascularizados, según extensión de la resección mandibular ${ }^{1,2,6}$.

- Cierre de comunicaciones bucosinusales con colgajo de bola adiposa de Bichat.

- Uso adicional de concentrados plaque$\operatorname{tarios}^{31}$.

- Biopsia diferida de secuestros óseos ${ }^{26}$.

- Valorar aporte de la suspensión de la terapia antiresortiva en cada caso ${ }^{9,17,26}$.
En los casos en que la patología se mantenga, a pesar de realizar los tratamientos anteriormente nombrados, se sugiere el uso de OHB como alternativa complementaria, teniendo en cuenta el bajo nivel de evidencia que existe sobre su uso ${ }^{27,29}$.

\section{Conclusiones}

La osteonecrosis de los maxilares (ONM) asociada a medicamentos es una patología recientemente reportada, con manifestaciones clínicas que pueden afectar significativamente la calidad de vida de los pacientes. A pesar de que su etiología no está del todo clara, debe ser siempre considerada como un riesgo en pacientes que requieran recibir drogas antiresortivas o antiangiogénicas.

La manera más efectiva de prevenir la ONM asociada a medicamentos es la evaluación odontológica exhaustiva, clínica e imaginológica, antes de iniciar la terapia. La mantención de una adecuada higiene y un buen estado de salud oral también son fundamentales. De ser posible, los tratamientos odontológicos conservadores deben privilegiarse por sobre los quirúrgicos, en pacientes con historia o tratamiento actual con medicamentos antiresortivos y/o antiangiogénicos.

Finalmente, es importante tener en consideración que cada paciente presenta una enfermedad de base e historia de terapias diferentes; por lo tanto, la toma de decisiones terapéuticas debe ser estudiada caso a caso de forma interdisciplinaria, por el equipo médico u oncológico tratante y el cirujano maxilofacial u odontólogo competente, con el fin de obtener mejores resultados y afectar lo menos posible la calidad de vida de los pacientes.

\section{Referencias}

1. Ruggiero SL, Dodson TB, Fantasia J, Goodday R, Aghaloo T, Mehrotra B, et al. American association of oral and maxillofacial surgeons position paper on medication-related osteonecrosis of the jaw - 2014 update. J Oral Maxillofac Surg 2014; 72 (10): 1938-1956. doi:10.1016/j.joms.2014.04.031.

2. Khan AA, Morrison A, Hanley DA, Felsenberg D, McCauley LK, O’Ryan F, et al. Diagnosis and management of osteonecrosis of the jaw: A systematic review and international consensus. J Bone Miner Res 2015; 30 (1): 3-23. doi:10.1002/jbmr.2405. 
3. Hess LM, Jeter JM, Benham-Hutchins M, Alberts DS. Factors Associated with Osteonecrosis of the Jaw among Bisphosphonate Users. Am J Med 2008; 121 (6). doi:10.1016/j.amjmed.2008.01.047.

4. Aghaloo TL, Dry SM, Mallya S, Tetradis S. Stage 0 osteonecrosis of the jaw in a patient on denosumab. J Oral Maxillofac Surg 2014; 72 (4): 702-16. doi:10.1016/j. joms.2013.09.008.

5. Hoff AO1, Toth BB, Altundag K, Johnson MM, Warneke CL, Hu M, et al. Frequency and risk factors associated with osteonecrosis of the jaw in cancer patients treated with intravenous bisphosphonates. J Bone Miner Res. 2008; 23 (6): 826-36. doi:10.1359/jbmr.080205.

6. Ruggiero SL, Dodson TB, Assael LA, Landesberg R, Marx RE, Mehrotra B. American Association of Oral and Maxillofacial Surgeons Position Paper on Bisphosphonate-Related Osteonecrosis of the Jaw - 2009 update. Aust Endod J 2009; 35 (3): 119-30. doi:10.1111/j.17474477.2009.00213.x.

7. Yamashita J, McCauley LK, Van Poznak C. Updates on osteonecrosis of the jaw. Curr Opin Support Palliat Care 2010; 4 (3): 200-6. doi:10.1097/SPC.0b013e32833d303b.

8. McLeod NMH, Brennan PA, Ruggiero SL. Bisphosphonate osteonecrosis of the jaw: A historical and contemporary review. Surgeon 2012; 10 (1): 36-42. doi: 10.1016/j.surge.2011.09.002.

9. Hellstein JW, Adler RA, Edwards B, Jacobsen PL, Kalmar JR, Koka S, et al. Managing the care of patients receiving antiresorptive therapy for prevention and treatment of osteoporosis: Executive summary of recommendations from the American Dental Association Council on Scientific Affairs. J Am Dent Assoc 2011; 142 (11): 1243-51. doi: 10.14219/jada.archive.2011.0108.

10. Woo S, Hellstein JW, Kalmar JR. Annals of Internal Medicine Review Systematic Review: Bisphosphonates and Osteonecrosis of the Jaws OF OF. Ann Intern Med. 2006; 144 (10): 753-761. doi: 10.7326/0003-4819-14410-200605160-00009.

11. Saad F, Brown JE, Van Poznak C, Ibrahim T, Stemmer $\mathrm{SM}$, Stopeck AT, et al. Incidence, risk factors, and outcomes of osteonecrosis of the jaw: Integrated analysis from three blinded active-controlled phase III trials in cancer patients with bone metastases. Ann Oncol 2012; 23 (5): 1341-7. doi: 10.1093/annonc/mdr435.

12. Wessel JH, Dodson TB, Zavras AI. Zoledronate, Smoking, and Obesity Are Strong Risk Factors for Osteonecrosis of the Jaw: A Case-Control Study. J Oral Maxillofac Surg 2008; 66 (4): 625-31. doi: 10.1016/j. joms.2007.11.032.

13. Bagán J, Blade J, Cozar JM, Constela M, García Sanz $\mathrm{R}$, Gómez Veiga F, et al. Recommendations for the prevention, diagnosis, and treatment of osteonecrosis of the jaw (ONJ) in cancer patients treated with bisphosphonates. Med Oral Patol Oral Cir Bucal 2007; 12 (4): 336-40.

14. Vassiliou V, Tselis N, Kardamakis D. Osteonecrosis of the jaws: Clinicopathologic and radiologic characteristics, preventive and therapeutic strategies. Strahlentherapie und Onkol 2010; 186 (7): 367-73. doi: 10.1007/ s00066-010-2066-9.

15. Fleisher KE, Chan KC. Amintavakoli N. Antiresorptive Drug-related Osteonecrosis of the Jaw (ARONJ)- a Guide to Research; 2016.

16. Fleisch H. Development of bisphosphonates. Breast Cancer Res 2002; 4 (1): 30-4. doi:10.1186/bcr414.

17. McClung M, Harris ST, Miller PD, Bauer DC, Davison KS, Dian L, et al. Bisphosphonate therapy for osteoporosis: Benefits, risks, and drug holiday. Am J Med. 2013; 126 (1): 13-20. doi: 10.1016/j.amjmed.2012.06.023.

18. Wei X1, Pushalkar S, Estilo C, Wong C, Farooki A, Fornier $\mathrm{M}$, et al. Molecular profiling of oral microbiota in jawbone samples of bisphosphonate-related osteonecrosis of the jaw. Oral Dis 2012; 18 (6): 602-12. doi: 10.1111/j.1601-0825.2012.01916.x.

19. Hoff AO, Toth BB, Altundag K, Guarneri V, Adamus A, NookaG AK, et al. Osteonecrosis of the jaw in patients receiving intravenous bisphosphonate therapy. ASCO Meet Abstr 2006; 24 (18_suppl): 8528. http://hwmaint. meeting.ascopubs.org/cgi/content/abstract/24/18_su$\mathrm{ppl} / 8528$.

20. Marx RE, Sawatari Y, Fortin M, Broumand V. Bisphosphonate-induced exposed bone (osteonecrosis/osteopetrosis) of the jaws: Risk factors, recognition, prevention, and treatment. J Oral Maxillofac Surg 2005; 63 (11): 1567-75. doi: 10.1016/j.joms.2005.07.010.

21. Marx RE, Tursun R. Suppurative osteomyelitis, bisphosphonate induced osteonecrosis, osteoradionecrosis: A blinded histopathologic comparison and its implications for the mechanism of each disease. Int J Oral Maxillofac Surg 2012; 41 (3): 283-9. doi: 10.1016/j. ijom.2011.12.016.

22. Wood J, Bonjean K, Ruetz S. Novel Antiangiogenic Effects of the Biphosphonate Compound Zolendronic Acid. J Assoc Off Anal Chem 2002; 302 (3): 1055-61. doi: 10.1124/jpet.102.035295.opment.

23. Fedele S, Porter SR, D'Aiuto F, Aljohani S, Vescovi P, Manfredi M, et al. Nonexposed variant of bisphosphonate-associated osteonecrosis of the jaw: A case series. Am J Med 2010; 123 (11): 1060-4. doi: 10.1016/j.amjmed.2010.04.033.

24. Barasch A, Cunha-Cruz J, Curro F, DeRouen T, Gilbert $\mathrm{GH}$, Hujoel P, et al. Dental risk factors for osteonecrosis 
of the jaws: A CONDOR case-control study. Clin Oral Investig 2013; 17 (8): 1839-45. doi: 10.1007/s00784-0120880-4.

25. Nh B, Mayer B, Hussein H, Zolk O. Interventions for managing medication-related osteonecrosis of the jaw (Review) Summary Of Findings For The Main Comparison 2017; (10). doi: 10.1002/14651858.CD012432. pub2.www.cochranelibrary.com.

26 Junquera LM, Martín-Granizo R. Diagnóstico, prevención y tratamiento de la osteonecrosis de los maxilares por bisfosfonatos. Recomendaciones de la Sociedad Española de Cirugía Oral y Maxilofacial (SECOM). Rev Esp Cir Oral y Maxilofac 2008; 30 (3): 145-56.

27. Freiberger JJ, Padilla-Burgos R, McGraw T, Suliman HB, Kraft KH, Stolp BW, et al. What is the role of hyperbaric oxygen in the management of bisphosphonate-related osteonecrosis of the jaw: A randomized controlled trial of hyperbaric oxygen as an adjunct to surgery and antibiotics. J Oral Maxillofac Surg 2012; 70 (7): 1573-83. doi:10.1016/j.joms.2012.04.001.

28. Klingelhöffer C, Zeman F, Meier J, Reichert TE, Ettl T. Evaluation of surgical outcome and influencing risk factors in patients with medication-related osteonecrosis of the jaws. J Cranio-Maxillofacial Surg 2016; 44 (10): 1694-9. doi: 10.1016/j.jcms.2016.08.001.

29. Al Hadi H, Smerdon GR, Fox SW. Hyperbaric oxygen therapy accelerates osteoblast differentiation and promotes bone formation. J Dent 2015; 43 (3): 382-8. doi: 10.1016/j.jdent.2014.10.006.

30. McLeod NMH, Patel V, Kusanale A, Rogers SN, Brennan PA. Bisphosphonate osteonecrosis of the jaw: A literature review of UK policies versus international policies on the management of bisphosphonate osteonecrosis of the jaw. Br J Oral Maxillofac Surg 2011; 49 (5): 335-42. doi:10.1016/j.bjoms.2010.08.005.
31. Lopez-Jornet P, Sanchez Perez A, Amaral Mendes R, Tobias A. Medication-related osteonecrosis of the jaw: Is autologous platelet concentrate application effective for prevention and treatment? A systematic review. J Cranio-Maxillofacial Surg 2016; 44 (8): 1067-72. doi: 10.1016/j.jcms.2016.05.004.

32. Delanian S, Chatel C, Porcher R, Depondt J, Lefaix JL. Complete restoration of refractory mandibular osteoradionecrosis by prolonged treatment with a pentoxifylline-tocopherol-clodronate combination (PENTOCLO): A phase II trial. Int J Radiat Oncol Biol Phys 2011; 80 (3): 832-9. doi: 10.1016/j.ijrobp.2010.03.029.

33. Patel V, Gadiwalla Y, Sassoon I, Sproat C, Kwok J, McGurk M. Prophylactic use of pentoxifylline and tocopherol in patients who require dental extractions after radiotherapy for cancer of the head and neck. Br J Oral Maxillofac Surg 2016; 54 (5): 547-50. doi: 10.1016/j. bjoms.2016.02.024.

34. Yalcin-Ulker GM, Cumbul A, Duygu-Capar G, Uslu Ü, Sencift K. Preventive Effect of Phosphodiesterase Inhibitor Pentoxifylline Against Medication-Related Osteonecrosis of the Jaw: An Animal Study. J Oral Maxillofac Surg 2017; 75 (11): 2354-68. doi: 10.1016/j. joms.2017.04.017.

35. Epstein MS, Wicknick FW, Epstein JB, Berenson JR, Gorsky M. Management of bisphosphonate-associated osteonecrosis: Pentoxifylline and tocopherol in addition to antimicrobial therapy. An initial case series. Oral Surgery, Oral Med Oral Pathol Oral Radiol Endodontology. 2010; 110 (5): 593-6. doi: 10.1016/j. tripleo.2010.05.067.

36. Dental A, Council A. Dental management of patients receiving oral bisphosphonate therapy: Expert panel recommendations. J Am Dent Assoc 2006; 137 (8): 1144-50. doi: 10.14219/jada.archive.2006.0355. 